\title{
The Diversity of Kilonova Emission in Short Gamma-Ray Bursts
}

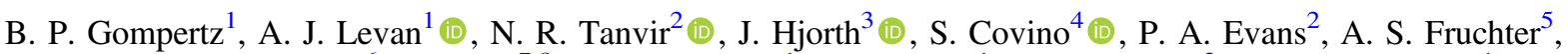 \\ C. González-Fernández ${ }^{6}$, Z. P. Jin ${ }^{7,8}$ (D) J. D. Lyman ${ }^{1}$, S. R. Oates ${ }^{1}$, P. T. O’Brien ${ }^{2}$, and K. Wiersema ${ }^{1}$ (D) \\ ${ }^{1}$ Department of Physics, University of Warwick, Coventry CV4 7AL, UK; b.gompertz@warwick.ac.uk \\ ${ }^{2}$ Department of Physics and Astronomy, University of Leicester, Leicester LE1 7RH, UK \\ ${ }^{3}$ Dark Cosmology Centre, Niels Bohr Institute, University of Copenhagen, Juliane Maries Vej 30, DK-2100 Copenhagen, Denmark \\ ${ }^{4}$ INAF, Osservatorio Astronomico di Brera, Via E. Bianchi 46, I-23807 Merate (LC), Italy \\ ${ }^{5}$ Space Telescope Science Institute, 3700 San Martin Drive, Baltimore, MD 21218, USA \\ ${ }^{6}$ Institute of Astronomy, University of Cambridge, Madingley Road, Cambridge CB3 OHA, UK \\ ${ }^{7}$ Key Laboratory of dark Matter and Space Astronomy, Purple Mountain Observatory, Chinese Academy of Science, Nanjing 210008, People's Republic of China \\ ${ }^{8}$ School of Astronomy and Space Science, University of Science and Technology of China, Hefei, Anhui 230026, People's Republic of China \\ Received 2017 October 12; revised 2018 April 13; accepted 2018 April 30; published 2018 June 12
}

\begin{abstract}
The historic first joint detection of both gravitational-wave and electromagnetic emission from a binary neutron star merger cemented the association between short gamma-ray bursts (SGRBs) and compact object mergers, as well as providing a well-sampled multi-wavelength light curve of a radioactive kilonova (KN) for the first time. Here, we compare the optical and near-infrared light curves of this KN, AT 2017gfo, to the counterparts of a sample of nearby $(z<0.5)$ SGRBs to characterize their diversity in terms of their brightness distribution. Although at similar epochs AT 2017gfo appears fainter than every SGRB-associated KN claimed so far, we find three bursts (GRBs 050509B, 061201, and 080905A) where, if the reported redshifts are correct, deep upper limits rule out the presence of a KN similar to AT 2017gfo by several magnitudes. Combined with the properties of previously claimed $\mathrm{KNe}$ in SGRBs this suggests considerable diversity in the properties of $\mathrm{KN}$ drawn from compact object mergers, despite the similar physical conditions that are expected in many NS-NS mergers. We find that observer angle alone is not able to explain this diversity, which is likely a product of the merger type (NS-NS versus NS$\mathrm{BH})$ and the detailed properties of the binary (mass ratio, spins etc.). Ultimately disentangling these properties should be possible through observations of SGRBs and gravitational-wave sources, providing direct measurements of heavy element enrichment throughout the universe.
\end{abstract}

Key words: gamma-ray burst: general - gravitational waves - nuclear reactions, nucleosynthesis, abundances stars: neutron

\section{Introduction}

Short gamma-ray bursts (SGRBs) have long been thought to be the products of the mergers of compact objects (Rosswog et al. 2003; Belczynski et al. 2006; Nakar 2007)—either binary neutron star (BNS) or neutron star-black hole (NS-BH) systems. In this framework, the energy release of the merger launches relativistic jets that produce $\gamma$-rays in internal shocks. A broadband synchrotron afterglow, with emission ranging from X-ray to radio frequencies, is then produced as the outflow decelerates in the circumstellar environment (Mészáros \& Rees 1993). Such a merger is also expected to produce a faint optical/near-IR (NIR) transient known as a "kilonova" (KN, or "macronova"; $\mathrm{Li} \&$ Paczyński 1998; Rosswog 2005; Metzger et al. 2010) as ejected material rich in neutrons forms heavy elements through rapid neutron capture ( $r$-process) nucleosynthesis (Lattimer \& Schramm 1974; Eichler et al. 1989; Freiburghaus et al. 1999) that subsequently decay radioactively. However, the discovery of GW170817 (LIGO Scientific \& Virgo Collaboration et al. 2017a; LIGO Scientific Collaboration 2017b) by the Advanced Laser Interferometer Gravitational-wave Observatory (LIGO) and Advanced Virgo provided the first direct evidence that the merger of a BNS has occurred. With it came the detection of SGRB 170817A by Fermi (Fermi-GBM 2017; Goldstein et al. 2017; von Kienlin et al. 2017) and later INTEGRAL (Savchenko et al. 2017). In broadband follow-up observations (LIGO Scientific Collaboration 2017b), an optical transient (Coulter et al. 2017a, 2017b; Soares-Santos et al. 2017) identified as a KN (AT 2017gfo; e.g., Cowperthwaite et al. 2017; Chornock et al. 2017; Evans et al. 2017; Nicholl et al. 2017; Pian et al. 2017; Smartt et al. 2017; Tanvir et al. 2017) was detected, thus confirming the compact object merger origin for SGRBs and $\mathrm{KNe}$.

Since 2005, the rapid localizations provided by the Swift satellite have meant that a catalog of SGRB afterglows at multiple wavelengths has been established. These afterglows are at their brightest just after the gamma-ray emission and decay away in the minutes to days following the burst. Interestingly, in GW170817, no afterglow was detected after the Fermi trigger. Despite being tied to NGC 4993, a nearby early-type galaxy at $z=0.009783$, or just $42.5 \mathrm{Mpc}$ (Blanchard et al. 2017; Hjorth et al. 2017; Levan et al. 2017), no X-ray emission was detected down to $2.7 \times 10^{-13} \mathrm{erg} \mathrm{s}^{-1} \mathrm{~cm}^{-2}$ at 0.62 days post-trigger (Evans et al. 2017). However, GRB jets are brightest on-axis, and are expected to be fainter and exhibit afterglows that rise later, as the observer moves off-axis. It is therefore tempting to ascribe the faint GRB (Fermi-GBM 2017; Goldstein et al. 201 von Kienlin et al. 2017) and slowly rising afterglow emission (Troja et al. 2017; Mooley et al. 2018; Lyman et al. 2018; Margutti et al. 2018) to an SGRB viewed away from the axis of its relativistic jet. Indeed, the GW data suggest a viewing angle of up to $28^{\circ}$ from the rotation axis of the binary (Evans et al. 2017; Haggard et al. 2017; LIGO Scientific Collaboration 2017b; Margutti et al. 2017; Tanvir et al. 2017; Mandel 2018), consistent with an off-axis model. However, the widely adopted simplification of a "top hat" jet, 
in which emission drops off sharply outside of narrow beam, likely needs to be modified to include structure away from the core of the jet (e.g., Lazzati et al. 2017). Alternative models featuring emission from a mildly relativistic cocoon of ejecta have also been proposed (e.g., Kasliwal et al. 2017b; Gottlieb et al. 2018; Mooley et al. 2018), though these too may feature a polar jet that could be detected as a more typical cosmological SGRB if it had been oriented toward the Earth.

Due to their typical discovery via detection of the $\gamma$-ray jet, SGRBs are normally viewed down the jet axis where the afterglow is brightest and therefore most likely to mask a $\mathrm{KN}$, which is expected to be a more isotropic component, that would normally be easier to see against the glare of the SGRB afterglow at angles away from the SGRB jet (Metzger \& Berger 2012). Nonetheless, candidate KNe have been observed in just a handful of events: the first and best claim coming with SGRB 130603B (Berger et al. 2013; Tanvir et al. 2013), followed by a reanalysis of SGRBs 060614 (Yang et al. 2015) and 050709 (Jin et al. 2016), and a so far inconclusive fourth candidate in SGRB 160821B (Jin et al. 2017; Kasliwal et al. 2017a).

Through association with BNS mergers and KNe, SGRBs are now thought to be the site of a sizable fraction of heavy element production in the universe (Lattimer \& Schramm 1974; Rosswog et al. 1998; Goriely et al. 2011; Korobkin et al. 2012; Just et al. 2015), supplementing the apparently insufficient yields predicted for supernovae (Thielemann et al. 2011). The identification of a clear $\mathrm{KN}$ signature accompanying GW170817 and GRB 170817A provides the perfect opportunity to assess their general detectability in SGRBs and the variability in their properties. Known BNS in the Milky Way exist in a rather small range of total mass and mass ratio (Lattimer 2011; Tauris et al. 2017), and so one might naively expect them to drive similar $\mathrm{KNe}$, which therefore ought to be reflected in the SGRB KN population if it is comprised entirely of BNS mergers. Alternatively, it might be that fine differences in the merging binaries or viewer orientation yield strongly differing observables, or that many SGRBs arise from NS-BH rather than BNS mergers.

In this paper, we compare the optical and near-infrared light curves of the KN in GW170817 to a sample of nearby $(z \lesssim 0.5)$ SGRBs to ascertain whether or not a $\mathrm{KN}$ of similar magnitude to AT 2017gfo could (or even should) have been detected. We use a cosmology of $H_{0}=67.8 \mathrm{~km} \mathrm{~s}^{-1} \mathrm{Mpc}^{-1}$, $\Omega_{M}=0.308$, and $\Omega_{\Lambda}=0.692$ (Planck Collaboration et al. 2016) throughout. All reported errors are $1 \sigma$, and given upper limits are $3 \sigma$.

\section{AT 2017gfo-The Kilonova Associated with GW170817}

Our data for AT 2017gfo come from Tanvir et al. (2017). We convert them to absolute magnitudes using a redshift of $z=0.009783$ (Hjorth et al. 2017; Levan et al. 2017).

To represent the evolution of AT 2017 gfo, we fit the light curves with Bazin functions (Bazin et al. 2011), which provide an estimate of rise $\left(\tau_{\text {rise }}\right)$ and decay $\left(\tau_{\text {fall }}\right)$ times by fitting the analytical function for a given band

$$
f(t)=A \frac{e^{\left.-\left(t-t_{0}\right)\right) / \tau_{\text {fall }}}}{1+e^{-\left(t-t_{0}\right) / \tau_{\text {rise }}}},
$$

where $A$ is the normalization. Bazin et al. (2011) also present an additive constant, $c$, which we find to be zero in each case. We therefore refit with the constant excluded. Our fits are

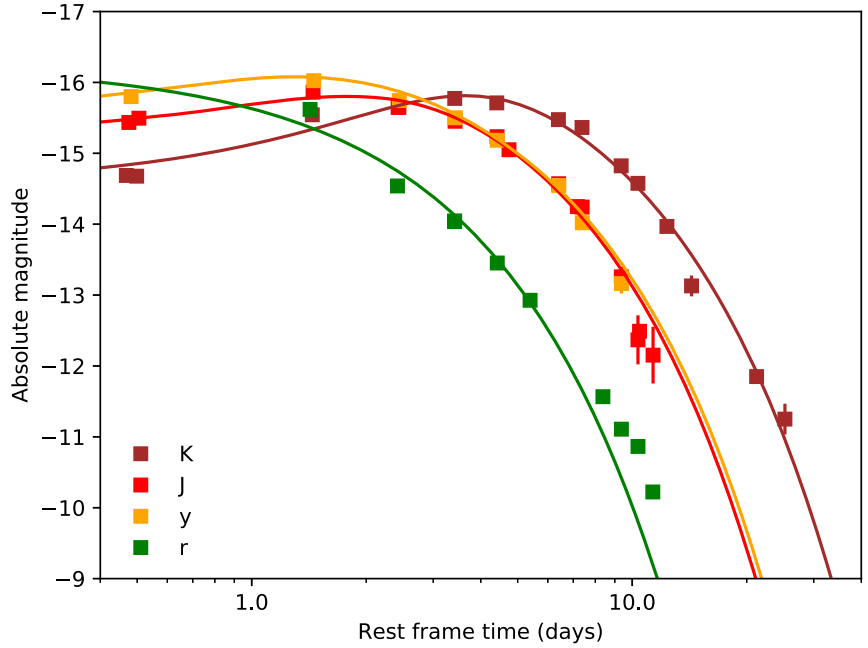

Figure 1. Model fit to AT 2017gfo. The $y, J$, and $K$ bands are fitted with a Bazin function, while the $r$ band is better fitted with a simple exponential. Error bars are smaller than the plot symbols in most cases.

Table 1

The Best Fits for AT 2017gfo, Taken in the Observer Frame

\begin{tabular}{lcccr}
\hline \hline Band & $\begin{array}{c}A \\
(\mu \mathrm{Jy})\end{array}$ & $\begin{array}{c}t_{0} \\
(\mathrm{hr})\end{array}$ & $\begin{array}{c}\tau_{\text {rise }} \\
(\mathrm{hr})\end{array}$ & $\begin{array}{r}\tau_{\text {fall }} \\
(\mathrm{hr})\end{array}$ \\
\hline$r$ & 544.50 & $\ldots$ & $\cdots$ & 42.27 \\
$y$ & 752.86 & 11.52 & 14.05 & 74.17 \\
$J$ & 652.96 & 24.87 & 20.00 & 71.01 \\
$K$ & 635.33 & 58.20 & 27.21 & 109.87 \\
\hline
\end{tabular}

Note. The $y, J$, and $K$ bands are fitted with a Bazin function (Equation (1)), while the $r$ band was better fitted with a simple exponential.

shown in Figure 1, while the fit parameters are given in Table 1 .

These fits demonstrate the strongly chromatic behavior of AT 2017gfo. Indeed, the $r$ band shows no apparent peak within the span of our data and declines from $\sim 1.5$ days as a simple exponential decay. This decay is very different to the power law seen in GRB afterglows and coupled with the nondetection of X-rays at comparable times rules out an afterglow component brighter than an $r$-band absolute magnitude of $M_{r} \sim-13$ at 0.62 days, assuming the mean $r$-band to X-ray flux ratio of 1130 found for SGRBs in Nysewander et al. (2009). Instead, the optical light is probably dominated by a rapidly fading transient created by the synthesis of lanthanidefree ejecta, with relatively low opacity (Evans et al. 2017). The redder bands are not well explained by such a simple model, and indeed the light curves appear progressively broader as the observations move redward, and can be explained by a lanthanide-rich, higher-opacity component (Tanvir et al. 2017).

\section{SGRB Data Sample}

We searched for nearby SGRBs with identified redshifts of $z<0.5$ in published works (Nysewander et al. 2009; Fong et al. 2015) and unpublished archives. ${ }^{9}$ For our sample, we define "short" GRBs as those with $T_{90}<2$ s (Kouveliotou et al. 1993), where $T_{90}$ is the duration of the middle $90 \%$ of the prompt emission fluence. However, we also include the population of

9 www.mpe.mpg.de/ jog/grbgen.html 
Table 2

The Sample of SGRBs with Their Redshifts, $z$, and the Source of Their Photometry

\begin{tabular}{llllll}
\hline \hline GRB & $z$ & References & GRB & $z$ & \multicolumn{1}{c}{ References } \\
\hline $050509 \mathrm{~B}$ & 0.2248 & $(1),(2),(3)$ & 090515 & 0.403 & $(28),(29),(30),(31),(32),(33)$ \\
050709 & 0.161 & $(4),(5),(6),(7)$ & $100206 \mathrm{~A}$ & 0.407 & $(26),(34),(35),(36),(37),(38),(39)$ \\
050724 & 0.257 & $(8),(9)$ & $100625 \mathrm{~A}$ & 0.452 & $(26),(40),(41),(42)$ \\
051210 & 0.114 & $(10),(11)$ & $130603 \mathrm{~B}$ & 0.356 & $(43),(44),(45),(46)$ \\
$060502 \mathrm{~B}$ & 0.287 & $(12),(13),(14)$ & $140903 \mathrm{~A}$ & 0.351 & $(47)$ \\
060614 & 0.125 & $(15)$ & $150101 \mathrm{~B}$ & 0.134 & $(48),(49)$ \\
061006 & 0.438 & $(16)$ & $150120 \mathrm{~A}$ & 0.46 & $(50),(51)$ \\
061201 & 0.084 & $(17)$ & $150424 \mathrm{~A}$ & 0.3 & $(52),(53),(54),(55),(56)$ \\
061210 & 0.41 & $(18),(19),(20)$ & $160624 \mathrm{~A}$ & 0.483 & $(57),(58),(59)$ \\
$070724 \mathrm{~A}$ & 0.457 & $(21),(22),(23)$ & $160821 \mathrm{~B}$ & 0.16 & $(60),(61)$ \\
071227 & 0.384 & $(16),(24),(25),(26)$ & $170428 \mathrm{~A}$ & 0.454 & $(62),(63),(64)$ \\
$080905 \mathrm{~A}$ & 0.1218 & $(26),(27)$ & $\ldots$ & $\cdots$ & $\cdots$
\end{tabular}

Note. (1) Castro-Tirado et al. (2005); (2) Hjorth et al. (2005a); (3) Bloom et al. (2006); (4) Fox et al. (2005); (5) Hjorth et al. (2005b); (6) Covino et al. (2006); (7) Jin et al. (2016); (8) Berger et al. (2005); (9) Malesani et al. (2007); (10) Blustin et al. (2005); (11) Berger \& Boss (2005); (12) Poole \& Troja (2006); (13) Price et al. (2006); (14) Rumyantsev et al. (2006); (15) Yang et al. (2015); (16) D'Avanzo et al. (2009); (17) Stratta et al. (2007); (18) Melandri et al. (2006); (19) Mirabal \& Halpern (2006); (20) Cenko et al. (2006); (21) Cenko et al. (2007); (22) Berger et al. (2009); (23) Kocevski et al. (2010); (24) D’Avanzo et al. (2007); (25) Berger et al. (2007); (26) Nicuesa Guelbenzu et al. (2012); (27) Rowlinson et al. (2010); (28) Morgan et al. (2009); (29) Updike et al. (2009); (30) Cucchiara et al. (2009); (31) Siegel \& Beardmore (2009); (32) McLeod \& Williams (2009); (33) Perley et al. (2009); (34) Leloudas et al. (2010); (35) Kuroda et al. (2010);(36) Marshall \& Krimm (2010); (37) Berger \& Chornock (2010); (38) Andreev et al. (2010); (39) Rumyantsev et al. (2010); (40) Naito et al. (2010); (41) Landsman \& Holland (2010); (42) Fong et al. (2013); (43) Tanvir et al. (2013); (44) Cucchiara et al. (2013); (45) de Ugarte Postigo et al. (2014); (46) Fong et al. (2014); (47) Troja et al. (2016a); (48) D’Avanzo et al. (2015); (49) Fong et al. (2016); (50) Chornock \& Fong (2015); (51) Chester \& D'Elia (2015); (52) Marshall \& Beardmore (2015); (53) Malesani et al. (2015); (54) Kann et al. (2015); (55) Butler et al. (2015); (56) Knust et al. (2017); (57) Kuroda et al. (2016); (58) Kong et al. (2016); (59) de Pasquale \& D’Ai (2016); (60) Jin et al. (2017); (61) Kasliwal et al. (2017a); (62) Kuin \& Beardmore (2017); (63) Bolmer et al. (2017); (64) Troja et al. (2017).

"extended emission" SGRBs. These bursts exhibit negligible spectral lags and hard spectra within the initial pulse(s) inside $2 \mathrm{~s}$ (in common with the SGRB population at large), followed by a softer, low-intensity tail that inflates $T_{90}$ to several tens of seconds (Norris \& Bonnell 2006; Norris et al. 2010). Our identified sample is shown in Table 2. Optical and IR photometric data are then collected from the published literature, or from GCN circulars. Any Vega magnitudes are converted to $\mathrm{AB}$, and corrections for Galactic absorption are performed following the maps of Schlafly \& Finkbeiner (2011) and using $R_{v}=3.1$ for the Milky Way. We do not make any corrections for extinction in the SGRB host galaxies.

In AT 2017gfo, it appears that the optical and IR light is dominated by the $\mathrm{KN}$, at least for several days after the merger, and possibly for longer. Any afterglow component is very weak (Evans et al. 2017). However, in SGRBs the situation is very different. It is likely that most SGRBs are viewed close to the jet axis (see, e.g., Ryan et al. 2015), and so show X-ray and optical afterglows. These afterglows probably dominate the light, at least for the first several days after the merger. However, the early, bright blue, and UV emission from AT 2017 gfo also suggests that such KN may contribute here. Therefore, it is desirable to estimate the likely afterglow contribution in the optical bands at these times. SGRB afterglows are well established as synchrotron phenomena, and so the X-ray data can be fairly simply extrapolated to optical frequencies. This provides a baseline estimate for the expected magnitude of the afterglow, which can be compared to the optical/NIR observations to check for any excesses that may be symptomatic of a $\mathrm{KN}$ (see the shaded regions in Figure 2), although it suffers from limitations due to the large lever-arm between X-ray and optical frequencies, over which the errors associated with the X-ray observations become significant.

Swift X-Ray Telescope (XRT) data are obtained for each SGRB from the $1 \mathrm{keV}$ flux density light curves on the UK
Swift Science Data Centre (UKSSDC) burst analyzer ${ }^{10}$ (Evans et al. 2007, 2009), except for SGRB 150101B, where we take the Chandra data from Fong et al. (2016) because the Swift light curve is dominated by the active galactic nucleus (AGN) in the host galaxy. Additional X-ray data from Chandra and/or XMM-Newton are added for SGRBs 050709 (Fox et al. 2005), 050724 (Grupe et al. 2006), 130603B (Fong et al. 2014), and 140903A (Troja et al. 2016a). We correct the Swift data for absorption using a ratio of (counts-to-flux unabsorbed)/ (counts-to-flux observed), which we obtain from the automatically generated late-time photon counting mode spectral fit in the XRT spectrum repository on the UKSSDC website. The flux densities are converted to $\mathrm{AB}$ magnitudes and extrapolated to $6260 \AA$ ( $r$ band) for easy comparison with the optical data. The assumed spectral indices of the extrapolation are $\beta=0.5$, which represents the case in which the synchrotron cooling break is above the X-ray frequency, and $\beta=1.0$, which implies the synchrotron cooling break is down near optical frequencies. Both of these assume that the electron energies follow a powerlaw distribution with an index of $p=2$, which is the theoretically expected value (Sari et al. 1998). In nature, measurements of spectral and temporal indices often imply that this distribution is steeper than $p=2$ (e.g., Curran et al. 2010), suggesting that a steeper spectral index would be needed. However, the synchrotron cooling break is typically measured to be somewhere close to X-ray frequencies rather than in the optical part of the spectrum (e.g., Gompertz et al. 2015), so flux densities bracketed by our two extrapolations provide a reasonable estimation of where the SGRB afterglow should occur.

We convert all apparent magnitudes to absolute magnitudes, assuming for a given band $k, k_{\mathrm{abs}}=k_{\mathrm{AB}}-5 \log _{10}\left(d_{L} / 10 \mathrm{pc}\right)+$ $2.5 \log _{10}(1+z)$. Observed times are divided by $(1+z)$ to put them in the rest frame. We do not correct for the redshift of the

\footnotetext{
${ }^{10}$ www.swift.ac.uk/burst_analyser/
} 



Figure 2. Our sample of SGRB afterglows compared to the model fits of AT 2017gfo. Colored circles are detections, and triangles are upper limits. These data are presented in their observer frame filters, and the AT $2017 \mathrm{gfo}$ models (colored lines) are shifted in frequency to match them using linear interpolation of the fits in Figure 1 supplemented by the $g$-band data from Tanvir et al. (2017) and the $u$-band data from Evans et al. (2017). We do not extrapolate if the rest-frame wavelength is below the $u$ band ( $3560 \AA$ ), so some data do not have models plotted. The gray band is the extrapolation of the X-ray flux to $6260 \AA$ ( $r$ band). SGRBs from Table 2 with no constraining observations are not included. 

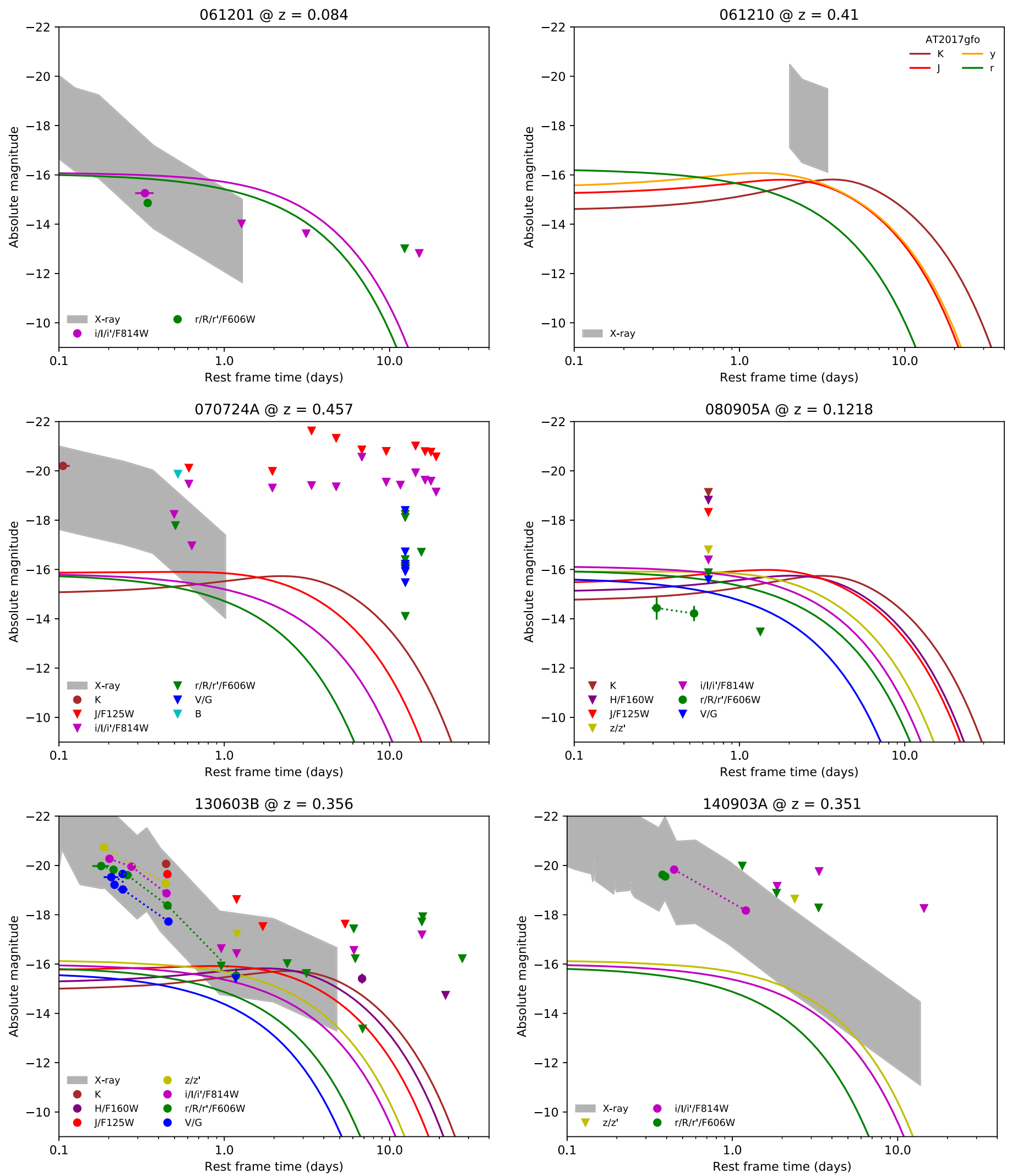

Figure 2. (Continued.) 

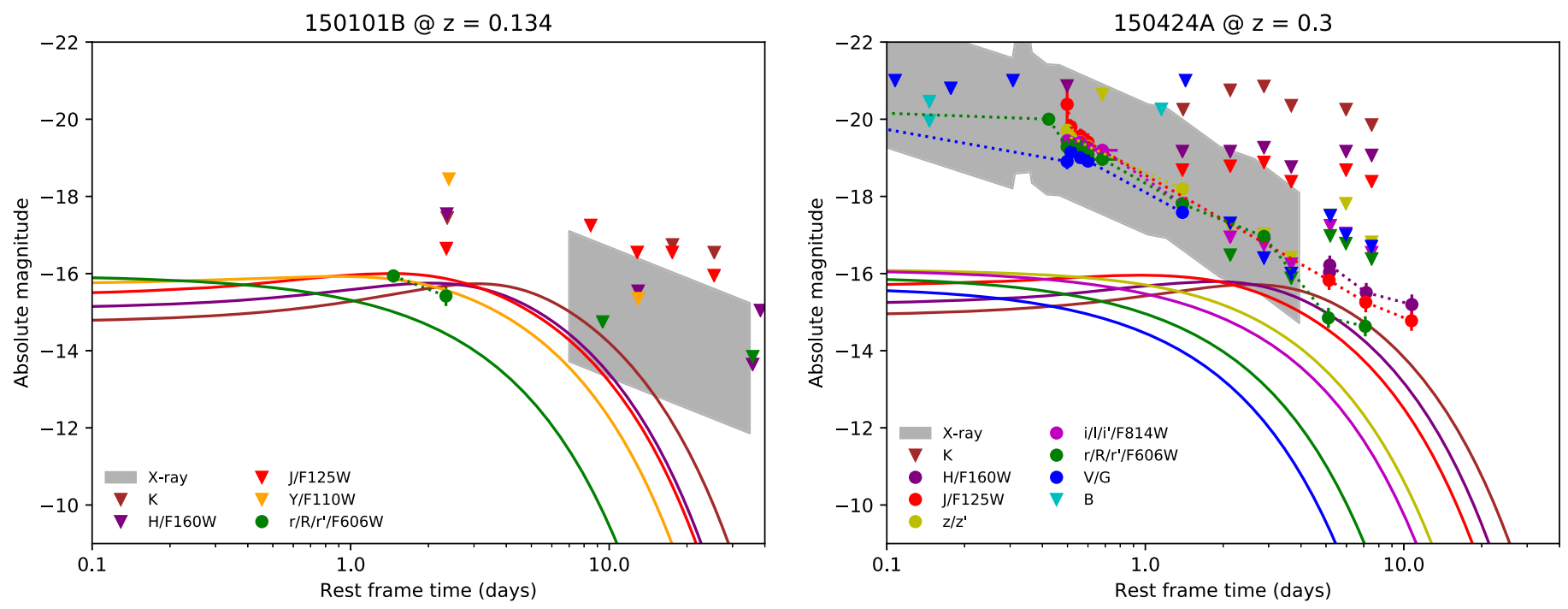

Figure 2. (Continued.)

central frequency of the photometric filter because this would require assuming a spectral shape that is uncertain in many SGRBs. Instead, we interpolate and shift the well-sampled light curve of AT 2017gfo to the rest-frame wavelength represented by the observations. This means our photometry is presented in its observer frame band, such that the magnitudes are given at $\lambda_{k}=(1+z) \lambda_{r}$, where $\lambda_{r}$ is the rest-frame emitting wavelength. Since KNe do not appear (or are at least very weak) at $\mathrm{X}$-ray frequencies, this issue does not affect our X-ray extrapolations.

While the optical-to-IR spectral energy distributions (SEDs) of our SGRBs are usually poorly constrained, we do have good color coverage of AT 2017 gfo. We therefore adjust our model fits to the wavelengths of the SGRB observations via linear interpolations between the fitted curves in Figure 1. Where the rest-frame frequency is blueward of the $r$-band fit to AT 2017 gfo, we supplement with observations with the SwiftUVOT $u$-band data from Evans et al. (2017) and the $H S T$ F475W ( $g$-band) data from Tanvir et al. (2017), with an early $g$-band point based on the spectral flux density measured in our MUSE spectroscopy. We fit both data sets with an exponential decay to ascertain the required color correction. Fitting in the observer frame, we find $A_{g}=321.92 \mu \mathrm{Jy}, \tau_{g \text {,fall }}=39.10 \mathrm{hr}$ and $A_{u}=321.39 \mu \mathrm{Jy}, \tau_{u \text {,fall }}=20.25 \mathrm{hr}$. We note that these bands have only a small number (3-4) of points to fit, and so we have assumed they follow an exponential decay as the $r$ band does, but do not have sufficient data to discriminate between alternative models. We do not extrapolate beyond the $u$ band if the rest-frame wavelength is less than $3560 \AA$.

\section{Results}

Light curves of our results are shown in Figure 2. Based on these light curves, our sample broadly divides into four main categories:

1. SGRBs with deep limits constraining to an AT 2017gfolike $\mathrm{KN}$.

2. SGRBs with candidate KNe.

3. SGRBs with afterglow detections bright enough to mask an AT 2017gfo-like KN.

4. SGRBs with no constraining observations.

\subsection{SGRBs with Deeper Limits than AT 2017gfo}

We find that for several SGRBs, a $\mathrm{KN}$ as bright as AT 2017 gfo could have been detected. For four bursts in particular, deep $3 \sigma$ upper limits that are a factor of two or more fainter than the detections of AT 2017gfo at comparable rest-frame times appear to rule out a KN-like AT 2017gfo. They are SGRBs 050509B (3 times fainter than AT 2017gfo), 051210 (2 times fainter), 061201 (4 times fainter), and 080905A (4.5 times fainter).

In each case, a KN similar to AT 2017gfo could have been detected if it had been present. These limits assume that in each case the redshift of the burst is correctly ascribed, and this is considered in more detail in Section 5. It should also be noted again that no host galaxy extinction has been included in our study.

\subsection{SGRBs with Candidate KNe}

Compared to the SGRB KN candidates, AT 2017gfo appears to be faint, though the contribution of the SGRB afterglow to the observed flux in the bursts is uncertain. The $H$-band detection in GRB 130603B is almost 3 times brighter than the interpolated $\mathrm{KN}$ model fit at the time of the observation. Similarly in SGRB 050709, the $K$-band detection is far brighter than the $K$ band of AT 2017 gfo. However, the $i$-band light curve appears to be fainter and peaks later, though the photometry is limited. The afterglow of GRB 060614 is already much brighter than the light curve of AT 2017gfo, and the $i$-band photometric excess (Yang et al. 2015) is over 50 times brighter than the contemporary $\mathrm{KN}$ fit, though it could be a later/longer rise like SGRB 050709. Alternatively, as shown in Figure 2, the extrapolation of the X-ray afterglow to optical/IR wavelengths may imply significant afterglow contributions at this time, such that the $\mathrm{KN}$ components can be fainter. The photometric excess claimed by Jin et al. (2017) in $160821 \mathrm{~B}$ is the only potential $\mathrm{KN}$ less bright than $\mathrm{AT}$ 2017gfo (see Troja et al. 2016b), though the data are inconclusive in this case (see N. R. Tanvir et al. 2018, in preparation for a detailed analysis of this burst). 


\subsection{SGRBs with Bright Afterglows}

We identify several SGRBs in which the afterglow was likely too bright for an AT 2017gfo-like KN to be detected. SGRB 150424A has optical/NIR afterglow detections several magnitudes brighter than AT 2017gfo (see Tanvir et al. 2015), and its detections and limits are never less than about 3 times brighter than the relevant model light curve in the $H$ band, or 10 times in the $r$ band. This burst may also be at a higher redshift than reported here (Tanvir et al. 2015), which would mean the afterglow is even brighter when corrected for distance. SGRBs 140903A and 150101B both have extrapolated X-ray afterglows that are brighter than AT 2017gfo, with optical detections that support the extrapolation, though there are no NIR limits constraining a redder transient. The $i$-band detection in SGRB 140903A is almost 15 times brighter than the AT 2017gfo model. The $r$-band detection in SGRB 150101B is just 2 times the flux of the AT 2017gfo model, so could potentially have a $\mathrm{KN}$ contribution. It is notable that its decay between the two epochs of observations is very similar to AT 2017 gfo. SGRB 050724 features a large flare seen in X-ray, NIR, and optical bands. The $i$-band detection close to 3 days is of a similar brightness to the one in 060614 (which was identified as a $\mathrm{KN}$ by its $i$-band excess; Yang et al. 2015), but the contribution from the various emission features is unknown.

\subsection{SGRBs with No Constraining Observations}

In the final category, six SGRBs do not have sufficiently deep observations to place any meaningful constraints on the presence of a KN-like AT 2017gfo.

SGRB 061210 may potentially have a bright afterglow if the extrapolated X-ray flux is in fact representative, but no observations in the optical/NIR are available. Neither SGRB 070724A nor 060502B have optical or NIR limits deep enough to provide meaningful constraints on the AT 2017 gfo models. However, the $r$-band limits in 060502B are within a factor of 2 of the $r$-band model of AT 2017gfo, suggesting that any $\mathrm{KN}$ in this burst is not significantly brighter than AT 2017gfo. SGRBs 061006, 071227, and 170428A all lie on bright host galaxies. No image subtraction of the host galaxy has been performed, and so any contributions from the afterglow or a possible $\mathrm{KN}$ are swamped by the light of the host.

\subsection{Colors}

One of the more distinct features of AT 2017gfo was its color evolution. The optical transient began blue, and slowly became redder over the course of several days (see, e.g., Figure 3 from Tanvir et al. 2017). This was explained as a two (or possibly three) component KN: a "blue" component from highvelocity lanthanide-poor dynamical ejecta from the poles (e.g., Nicholl et al. 2017), and a slower "red" lanthanide-rich component driven in an isotropic wind, and rising to prominence later. Some groups also invoke an intermediate "purple" component (e.g., Villar et al. 2017).

Many of the SGRBs in our sample feature multi-color detections, and this allows us to compare their colors to AT 2017gfo. Seven feature photometric detections in more than one filter that are approximately contemporaneous. They are SGRBs 050709, 050724, 060614, 061201, 130603B, 140903A and $150424 \mathrm{~A}$. We compare their colors to our model curves of
AT 2017gfo. Colors are compared in the rest frame, and so are $k$-corrected from their initial redshift.

From the KN candidates, SGRB 050709 appears to be bluer than AT 2017 gfo at around 2 rest-frame days after trigger when measured in $g-r(0.55 \pm 0.12$ in the SGRB data versus $\sim 0.96$ in our AT 2017gfo model) and $r-i(-0.30 \pm 0.22$ versus $\sim 0.50$ ). However, at around 5 days after trigger its $I-K$ color of $2.96 \pm 0.70$ is comparable within errors to our model fits, where $I-K \sim 2.12$. Whether this is due to color evolution is uncertain because we have no $K$-band magnitude measurements from the earlier epoch. At 2 days, SGRB 050709 is brighter in $r$ than in either $g$ or $i$. This is unusual for an SGRB afterglow, and potentially suggests some spectral evolution. SGRB 060614 is also bluer than AT 2017gfo early on, with $g-r=0.15 \pm 0.04$ in the data compared to $\sim 0.74$ in the model, and $r-i=0.05 \pm 0.04$ in the data versus $\sim 0.42$ in the model at around 1.5 rest-frame days. It shows a redward linear evolution in both colors in a similar way to AT $2017 \mathrm{gfo}$, becoming $g-r=0.30 \pm 0.10 \quad(\sim 1.31)$ and $r-i=0.41 \pm 0.11 \quad(\sim 0.56)$ over the next two days. By 2 weeks after trigger, the colors have converged, with $r-i=1.23 \pm 0.18$ in the data and $r-i$ $\sim 1.11$ in the model. This may indicate that the KN in 060614 now dominates the observed emission. Multi-wavelength coverage for SGRB 130603B is only available inside one day after trigger. This emission has already been shown to be consistent with an afterglow by Fong et al. (2014). The $r-i$ color is $0.45 \pm 0.06$, compared to $\sim 0.19$ for the contemporaneous AT 2017gfo model at 0.2 days.

At almost half a day after trigger, the $r-i$ color of the model is $\sim 0.25$, and three of the remaining four SGRBs with color information are broadly consistent with this: 061201 has $r-i$ $0.40 \pm 0.14 ; 140903 \mathrm{~A}$ has $r-i=0.28 \pm 0.08$; and $150424 \mathrm{~A}$ has $r-i=0.16 \pm 0.14$. The exception, 050724, exhibits a large flare at this time (Figure 2). There is no evidence for significant evolution beyond this time, in most cases due to a lack of continuing multi-color monitoring. SGRB 150424A has $r-i=0.01 \pm 0.15$ at 1.4 days, consistent with no evolution, while the AT 2017gfo model has evolved to $r-i \sim 0.65$ at this time. Jin et al. (2017) also found this SGRB to be consistent with no chromatic evolution.

\section{Discussion}

We search our sample of 23 SGRBs with an identified redshift of $z<0.5$ and optical/NIR observations for detections and limits constraining KNe similar to AT 2017gfo, or bright afterglows capable of masking a $\mathrm{KN}$ of this magnitude. Three have claimed $\mathrm{KNe}$ in the literature $(050709,060614,130603 \mathrm{~B})$ with a further marginal case (160821B; see N. R. Tanvir et al. 2018 , in preparation, for a detailed analysis). Four have limits deeper than AT 2017gfo (050509B, 051210, 061201, $080905 \mathrm{~A})$, and three of these are over a magnitude deeper (050509B, 061201, 080905A). One more has limits of comparable depth (060502B). Four have bright optical/NIR detections consistent with light arising from an afterglow component (050724, 140903A, 150101B, 150424A), although at least in one case at magnitudes only marginally brighter than AT 2017gfo (GRB 150101B). Two have afterglows that are implied to be bright by the X-ray extrapolation (061210, 070724A), although were in fact not observed (in the case of 070724A, the absence of the afterglow has been suggested to be due to the presence of dust; Berger et al. 2009). Three have bright host galaxies (061006, 071227, 170428A), and the 
remaining five are completely unconstrained by the available observations. The broad range of magnitudes in the sample suggests a diversity in the brightness of $\mathrm{KNe}$ associated with SGRBs.

For three bursts in particular, the absence of a $\mathrm{KN}$ is conspicuous. SGRBs 050509B, 061201, and 080905A all have limits much deeper than the detections for AT 2017gfo, and any $\mathrm{KN}$ would have had to have been at least five times fainter to be missed, yet AT 2017gfo itself is fainter than any KN seen in SGRBs so far. Our findings for SGRB 050509B are consistent with Fong et al. (2017).

There are two concerns that should be addressed in interpreting these limits. The first is whether there could be some unseen extinction along the line of sight. In the case of SGRBs 050509B and 051210 this is of particular concern because there is no optical light at any epoch and too few X-ray photons to determine a column density. However, SGRB 050509B is spatially coincident with the outskirts of a giant elliptical galaxy (Castro-Tirado et al. 2005; Gehrels et al. 2005; Hjorth et al. 2005b; Bloom et al. 2006), which is the putative host (and the source of the redshift). Given its location, it is unlikely that dust extinction plays a significant role. The apparent faintness of the optical/NIR emission is therefore more likely to be intrinsic, perhaps due to a sparse local environment or a wide viewing angle from the SGRB jet. For SGRBs 061201 and 080905A the detection of the sources in optical light rules out extreme extinction, but does not discount moderate levels. Fong et al. (2015) find that both of these bursts are consistent with having no host extinction in their modeling, and this suggests that the limits on a $\mathrm{KN}$ are indeed as constraining as they appear to be. It is notable in these examples that the optical light is consistent with the X-ray extrapolation (where available) with $\beta=0.5$, and so the bursts are not "dark" GRBs (Jakobsson et al. 2004).

The second concern is the validity of the assumed redshifts. Only one short GRB has a redshift measured in absorption, GRB 130603B (de Ugarte Postigo et al. 2014). In other cases, the redshifts are based on putative host galaxy identifications. For GRB 050509B there is a large $\mathrm{cD}$ galaxy close to the location and a coincidence with a massive, merging galaxy cluster. Much of the mass along this line of sight lies within this cluster, and the redshift has high confidence (see, e.g., Bloom et al. 2002; Levan et al. 2007). For GRB 080905A the burst position overlaps the spiral arm of the host galaxy, again suggesting a chance alignment probability of $\lesssim 1 \%$. However, for GRB 061201 the situation is more complex. The burst belongs to the so-called hostless SGRB population (Berger 2010; Tunnicliffe et al. 2014), and so the redshift is based on a proximity to the Abell 995 cluster (Berger 2006; Stratta et al. 2007). This is a rich cluster, but there are no galaxies within a few arcseconds of the burst position, and so the probability of chance alignment is significant, and the redshift should be viewed with caution. While the probabilities of any given burst redshift being wrong appear small, it should also be noted that assigning redshifts by probabilistic arguments favors the brightest nearby galaxies, many of which are likely to be closer, and so this may produce a bias in which incorrect host assignments lead to lower redshifts, and hence strong $\mathrm{KN}$ constraints.

The SGRBs with bright afterglows still may have KN contributions in the detected flux. The inferred contribution from an AT 2017gfo-like KN ranges from 1/15 (140903A,

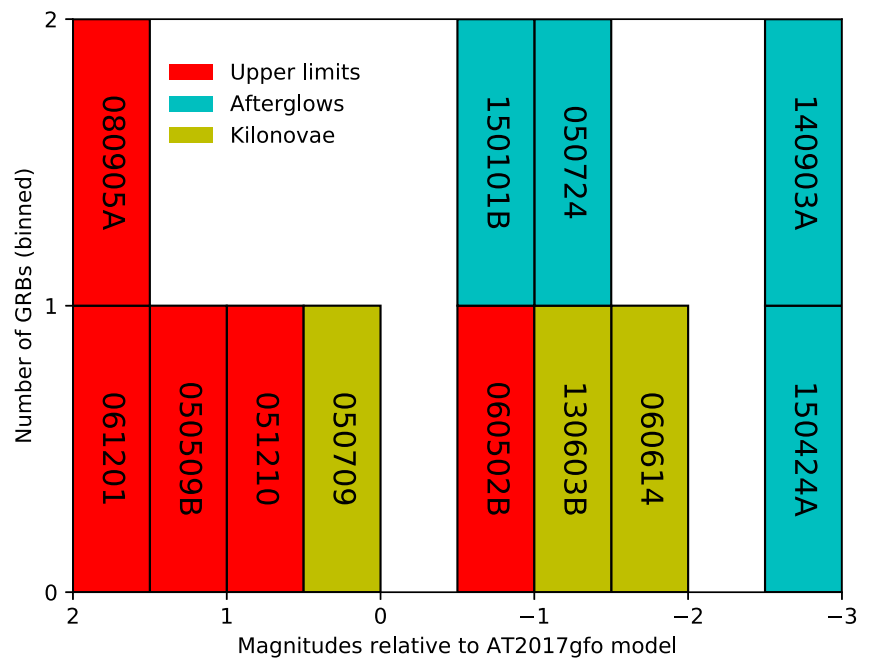

Figure 3. Snapshot of the $i$ - $/ r$-band magnitudes of our SGRB observations relative to the AT 2017 gfo models, taken between 0.5 and 3 days after trigger. Data are binned into 0.5 mag intervals. Bursts with data not constraining to the AT 2017 gfo models are not included. "Upper limits" (red) refers to bursts with deep limits constraining to an AT 2017gfo-like KN. "Afterglows" (blue) are the SGRBs with afterglows bright enough to mask AT 2017gfo. "Kilonovae" (yellow) are the SGRB KN candidates.

$i$ band) to $1 / 2.5$ (150101B, $r$ band), and the latter end of the scale is certainly enough to cause an SED to deviate away from the power law expected from the synchrotron afterglow. A KN was discovered this way for both SGRBs 050709 (Jin et al. 2016) and 060614 (Yang et al. 2015) and potentially 160821B as well (Jin et al. 2017). However, no multi-color observations are available for any of these bursts except 150424A, the brightest of the four. Jin et al. (2017) found no evidence of chromatic deviation in this source.

AT 2017gfo appears to be somewhat fainter than other established KN, but the lack of X-ray afterglow to deep limits $\left(2.7 \times 10^{-13} \mathrm{erg} \mathrm{s}^{-1} \mathrm{~cm}^{-2}\right.$ at 0.62 days after trigger; Evans et al. 2017) and constraints from the GW data suggest that the viewing angle is further off-axis than in typical SGRBsperhaps up to $28^{\circ}$ away from the jet axis (Evans et al. 2017; Haggard et al. 2017; LIGO Scientific Collaboration 2017b; Margutti et al. 2017; Tanvir et al. 2017). The fainter KN in GW170817 is therefore within the variation expected from the observer position (e.g., Grossman et al. 2014). The peak time and luminosity of a $\mathrm{KN}$ is also a function of ejecta mass and opacity (e.g., Metzger et al. 2010), and naturally some variation is expected from case to case.

\section{Conclusions}

Our analysis reveals a diverse range of $\mathrm{KN}$ possibilities, as in some SGRBs we find upper limits for optical/NIR emission several magnitudes deeper than AT 2017gfo, in others there are identified (or suspected) KNe that are brighter, and we also find SGRBs with bright afterglows capable of masking KNe that are brighter still. Our sample spans five magnitudes at a few days after trigger (Figure 3). The most interesting comparison is between the SGRBs with detected KNe (including AT 2017gfo) and the three SGRBs with deep limits; the diversity between these two groups is hard to reconcile with the highly uniform distribution of known BNS masses and mass ratios in the Milky Way (Lattimer 2011; Tauris et al. 2017). 
The relatively small range of viewing angles in the SGRB population means that the observer position alone probably cannot explain the $\sim 3.5 \mathrm{mag}$ (a factor of 25 in flux) between the KN in SGRB 060614 and the upper limits in SGRBs 061201 and $080905 \mathrm{~A}$. If their redshifts are correct, they may potentially suggest a BNS/NS-BH dichotomy in the SGRB population, as this represents the most natural way to explain an apparent contrast in the ejected masses available to power a $\mathrm{KN}$; an NS-BH merger can produce as much as 10 times more dynamical ejecta than can a BNS (Metzger 2017).

The LIGO/Virgo detection of GW170817 and electromagnetic follow-up and identification of SGRB 170717A and AT 2017gfo brings about the advent of KN astronomy. Further observations of $\mathrm{KNe}$ will reveal whether the magnitude of the emission forms a continuum or continues to display a gap in brightness between two populations. Unsurprisingly, our best constraints come from the SGRBs at the lowest redshifts, and our work emphasizes the need to perform $\mathrm{KN}$ searches at low $z$ and in NIR filters.

We thank the anonymous referee for useful comments that improved the manuscript.

B.G., A.J.L., N.R.T., and K.W. have received funding from the European Research Council (ERC) under the European Union's Horizon 2020 research and innovation programme (grant agreement No. 725246, TEDE; PI: Levan).

A.J.L. and J.D.L. acknowledge support from STFC via grant ST/P000495/1.

N.R.T. and K.W. acknowledge support from STFC via grant ST/N000757/1.

J.H. was supported by a VILLUM FONDEN Investigator grant (project number 16599).

S.C. acknowledges partial funding from Agenzia Spaziale Italiana-Istituto Nazionale di Astrofisica grant I/004/11/3.

S.R.O. gratefully acknowledges the support of the Leverhulme Trust Early Career Fellowship.

This work made use of data supplied by the UK Swift Science Data Centre at the University of Leicester.

\section{ORCID iDs}

A. J. Levan (i) https://orcid.org/0000-0001-7821-9369

N. R. Tanvir (1) https://orcid.org/0000-0003-3274-6336

J. Hjorth (1) https://orcid.org/0000-0002-4571-2306

S. Covino (1) https://orcid.org/0000-0001-9078-5507

Z. P. Jin (1) https://orcid.org/0000-0003-4977-9724

K. Wiersema (i) https://orcid.org/0000-0002-9133-7957

\section{References}

Andreev, M., Sergeev, A., Parakhin, N., et al. 2010, GCN, 10455, 1 Berger, E. 2006, GCN, 5952, 1

Bazin, G., Ruhlmann-Kleider, V., Palanque-Delabrouille, N., et al. 2011, A\&A, 534, A43

Belczynski, K., Perna, R., Bulik, T., et al. 2006, ApJ, 648, 1110

Berger, E. 2010, ApJ, 722, 1946

Berger, E., \& Boss, A. 2005, GCN, 4323, 1

Berger, E., Cenko, S. B., Fox, D. B., \& Cucchiara, A. 2009, ApJ, 704, 877

Berger, E., \& Chornock, R. 2010, GCN, 10410, 1

Berger, E., Fong, W., \& Chornock, R. 2013, ApJL, 774, L23

Berger, E., Morrell, N., \& Roth, M. 2007, GCN, 7151, 1

Berger, E., Price, P. A., Cenko, S. B., et al. 2005, Natur, 438, 988

Blanchard, P. K., Berger, E., Fong, W., et al. 2017, ApJL, 848, L22

Bloom, J. S., Kulkarni, S. R., \& Djorgovski, S. G. 2002, AJ, 123, 1111

Bloom, J. S., Prochaska, J. X., Pooley, D., et al. 2006, ApJ, 638, 354
Blustin, A. J., Mangano, V., Voges, W., Marshall, F., \& Gehrels, N. 2005, GCN, 4331, 1

Bolmer, J., Steinle, H., \& Schady, P. 2017, GCN, 21050, 1

Butler, N., Watson, A. M., Kutyrev, A., et al. 2015, GCN, 17762, 1

Castro-Tirado, A. J., de Ugarte Postigo, A., Gorosabel, J., et al. 2005, A\&A, 439, L15

Cenko, S. B., Fox, D. B., \& Price, P. A. 2006, GCN, 5912, 1

Cenko, S. B., Rau, A., Berger, E., Price, P. A., \& Cucchiara, A. 2007, GCN, 6664, 1

Chester, M. M., \& D'Elia, V. 2015, GCN, 17323, 1

Chornock, R., Berger, E., Kasen, D., et al. 2017, ApJL, 848, L19

Chornock, R., \& Fong, W. 2015, GCN, 17358, 1

Coulter, D. A., Foley, R. J., Kilpatrick, C. D., et al. 2017b, Sci, 358, 1556

Coulter, D. A., Kilpatrick, C. D., Siebert, M. R., et al. 2017a, GCN, 21529, 1

Covino, S., Malesani, D., Israel, G. L., et al. 2006, A\&A, 447, L5

Cowperthwaite, P. S., Berger, E., Villar, V. A., et al. 2017, ApJL, 848, L17

Cucchiara, A., Fox, D. B., Tanvir, N., et al. 2009, GCN, 9362, 1

Cucchiara, A., Prochaska, J. X., Perley, D., et al. 2013, ApJ, 777, 94

Curran, P. A., Evans, P. A., de Pasquale, M., Page, M. J., \& van der Horst, A. J. 2010, ApJL, 716, L135

D'Avanzo, P., D'Elia, V., Lorenzi, V., et al. 2015, GCN, 17326, 1

D'Avanzo, P., Malesani, D., Covino, S., et al. 2009, A\&A, 498, 711

D'Avanzo, P., Piranomonte, S., Antonelli, L. A., et al. 2007, GCN, 7149, 1

de Pasquale, M., \& D'Ai, A. 2016, GCN, 19576, 1

de Ugarte Postigo, A., Thöne, C. C., Rowlinson, A., et al. 2014, A\&A, 563, A62

Eichler, D., Livio, M., Piran, T., \& Schramm, D. N. 1989, Natur, 340, 126

Evans, P. A., Beardmore, A. P., Page, K. L., et al. 2007, A\&A, 469, 379

Evans, P. A., Beardmore, A. P., Page, K. L., et al. 2009, MNRAS, 397, 1177

Evans, P. A., Cenko, S. B., Kennea, J. A., et al. 2017, Sci, 358, 1565

Fermi-GBM 2017, GCN, 524666471, 1

Fong, W., Berger, E., Blanchard, P. K., et al. 2017, ApJL, 848, L23

Fong, W., Berger, E., Chornock, R., et al. 2013, ApJ, 769, 56

Fong, W., Berger, E., Margutti, R., \& Zauderer, B. A. 2015, ApJ, 815, 102

Fong, W., Berger, E., Metzger, B. D., et al. 2014, ApJ, 780, 118

Fong, W., Margutti, R., Chornock, R., et al. 2016, ApJ, 833, 151

Fox, D. B., Frail, D. A., Price, P. A., et al. 2005, Natur, 437, 845

Freiburghaus, C., Rosswog, S., \& Thielemann, F.-K. 1999, ApJL, 525, L121

Gehrels, N., Sarazin, C. L., O'Brien, P. T., et al. 2005, Natur, 437, 851

Goldstein, A., Veres, P., Burns, E., et al. 2017, ApJL, 848, L14

Gompertz, B. P., van der Horst, A. J., O'Brien, P. T., Wynn, G. A., \& Wiersema, K. 2015, MNRAS, 448, 629

Goriely, S., Bauswein, A., \& Janka, H.-T. 2011, ApJL, 738, L32

Gottlieb, O., Nakar, E., \& Piran, T. 2018, MNRAS, 473, 576

Grossman, D., Korobkin, O., Rosswog, S., \& Piran, T. 2014, MNRAS, 439,757

Grupe, D., Burrows, D. N., Patel, S. K., et al. 2006, ApJ, 653, 462

Haggard, D., Nynka, M., Ruan, J. J., et al. 2017, ApJL, 848, L25

Hjorth, J., Levan, A. J., Tanvir, N. R., et al. 2017, ApJL, doi:10.3847/2041$8213 /$ aa9110

Hjorth, J., Sollerman, J., Gorosabel, J., et al. 2005a, ApJL, 630, L117

Hjorth, J., Watson, D., Fynbo, J. P. U., et al. 2005b, Natur, 437, 859

Jakobsson, P., Hjorth, J., Fynbo, J. P. U., et al. 2004, ApJL, 617, L21

Jin, Z.-P., Hotokezaka, K., Li, X., et al. 2016, NatCo, 7, 12898

Jin, Z.-P., Li, X., Wang, H., et al. 2017, arXiv:1708.07008

Just, O., Bauswein, A., Pulpillo, R. A., Goriely, S., \& Janka, H.-T. 2015, MNRAS, 448, 541

Kann, D. A., Tanga, M., \& Greiner, J. 2015, GCN, 17757, 1

Kasliwal, M. M., Korobkin, O., Lau, R. M., Wollaeger, R., \& Fryer, C. L. 2017a, ApJL, 843, L34

Kasliwal, M. M., Nakar, E., Singer, L. P., et al. 2017b, Sci, 358, 1559

Knust, F., Greiner, J., van Eerten, H. J., et al. 2017, A\&A, 607, A84

Kocevski, D., Thöne, C. C., Ramirez-Ruiz, E., et al. 2010, MNRAS, 404, 963

Kong, A. K. H., Lee, M. Y., Lin, Y.-M., Hou, X., \& Liu, C. Y. 2016, GCN, 19575,1

Korobkin, O., Rosswog, S., Arcones, A., \& Winteler, C. 2012, MNRAS, 426, 1940

Kouveliotou, C., Meegan, C. A., Fishman, G. J., et al. 1993, ApJL, 413, L101

Kuin, N. P. M., \& Beardmore, A. P. 2017, GCN, 21049, 1

Kuroda, D., Hanayama, H., Miyaji, T., et al. 2016, GCN, 19571, 1

Kuroda, D., Yanagisawa, K., Shimizu, Y., et al. 2010, GCN, 10388, 1

Landsman, W., \& Holland, S. 2010, GCN, 10892, 1

Lattimer, J. M. 2011, Ap\&SS, 336, 67

Lattimer, J. M., \& Schramm, D. N. 1974, ApJL, 192, L145

Lazzati, D., Perna, R., Morsony, B. J., et al. 2017, arXiv:1712.03237

Leloudas, G., Xu, D., Malesani, D., et al. 2010, GCN, 10387, 1 
Levan, A. J., Jakobsson, P., Hurkett, C., et al. 2007, MNRAS, 378, 1439

Levan, A. J., Lyman, J. D., Tanvir, N. R., et al. 2017, ApJL, 848, L28

Li, L.-X., \& Paczyński, B. 1998, ApJL, 507, L59

LIGO Scientific \& Virgo Collaboration et al. 2017a, GCN, 21505, 1

LIGO Scientific Collaboration, Virgo Collaboration \& Partner Astronomy Groups 2017b, PhRvL, 119, 161101

Lyman, J. D., Lamb, G. P., Levan, A. J., et al. 2018, arXiv:1801.02669

Malesani, D., Covino, S., D’Avanzo, P., et al. 2007, A\&A, 473, 77

Malesani, D., Xu, D., Watson, D. J., \& Blay, P. 2015, GCN, 17756, 1

Mandel, I. 2018, ApJL, 853, L12

Margutti, R., Alexander, K. D., Xie, X., et al. 2018, arXiv:1801.03531

Margutti, R., Berger, E., Fong, W., et al. 2017, ApJL, 848, L20

Marshall, F. E., \& Beardmore, A. P. 2015, GCN, 17751, 1

Marshall, F. E., \& Krimm, H. A. 2010, GCN, 10394, 1

McLeod, B., \& Williams, G. 2009, GCN, 9370, 1

Melandri, A., Carter, D., Mundell, C., et al. 2006, GCN, 5920, 1

Mészáros, P., \& Rees, M. J. 1993, ApJ, 405, 278

Metzger, B. D. 2017, LRR, 20, 3

Metzger, B. D., \& Berger, E. 2012, ApJ, 746, 48

Metzger, B. D., Martínez-Pinedo, G., Darbha, S., et al. 2010, MNRAS, 406, 2650

Mirabal, N., \& Halpern, J. P. 2006, GCN, 5906, 1

Mooley, K. P., Nakar, E., Hotokezaka, K., et al. 2018, Natur, 554, 207

Morgan, A. N., Cobb, B. E., Klein, C., \& Bloom, J. S. 2009, GCN, 9359, 1

Naito, H., Sako, T., Suzuki, D., et al. 2010, GCN, 10889, 1

Nakar, E. 2007, PhR, 442, 166

Nicholl, M., Berger, E., Kasen, D., et al. 2017, ApJL, 848, L18

Nicuesa Guelbenzu, A., Klose, S., Greiner, J., et al. 2012, A\&A, 548, A101

Norris, J. P., \& Bonnell, J. T. 2006, ApJ, 643, 266

Norris, J. P., Gehrels, N., \& Scargle, J. D. 2010, ApJ, 717, 411

Nysewander, M., Fruchter, A. S., \& Pe'er, A. 2009, ApJ, 701, 824

Perley, D. A., Kislak, M., \& Ganeshalingam, M. 2009, GCN, 9372, 1

Pian, E., D’Avanzo, P., Benetti, S., et al. 2017, Natur, 551, 67

Planck Collaboration, Ade, P. A. R., Aghanim, N., et al. 2016, A\&A, 594, A13
Poole, T. S., \& Troja, E. 2006, GCN, 5069, 1

Price, P. A., Berger, E., Fox, D. B., Cenko, S. B., \& Rau, A. 2006, GCN, 5077,1

Rosswog, S. 2005, ApJ, 634, 1202

Rosswog, S., Ramirez-Ruiz, E., \& Davies, M. B. 2003, MNRAS, 345, 1077

Rosswog, S., Thielemann, F. K., Davies, M. B., Benz, W., \& Piran, T. 1998, in

Proc. of the 9th Workshop on Nuclear Astrophysics, ed. W. Hillebrandt \&

E. Muller (Garching bei München: Max Planck Institute for Astrophysics), 103

Rowlinson, A., Wiersema, K., Levan, A. J., et al. 2010, MNRAS, 408, 383

Rumyantsev, V., Karimov, R., Salyamov, R., et al. 2006, GCN, 5184, 1

Rumyantsev, V., Shakhovkoy, D., \& Pozanenko, A. 2010, GCN, 10456, 1

Ryan, G., van Eerten, H., MacFadyen, A., \& Zhang, B.-B. 2015, ApJ, 799, 3

Sari, R., Piran, T., \& Narayan, R. 1998, ApJL, 497, L17

Savchenko, V., Ferrigno, C., Kuulkers, E., et al. 2017, ApJ, 848, 15

Schlafly, E. F., \& Finkbeiner, D. P. 2011, ApJ, 737, 103

Siegel, M. H., \& Beardmore, A. P. 2009, GCN, 9369, 1

Smartt, S. J., Chen, T.-W., Jerkstrand, A., et al. 2017, Natur, 551, 75

Soares-Santos, M., Holz, D. E., Annis, J., et al. 2017, ApJL, 848, L16

Stratta, G., D'Avanzo, P., Piranomonte, S., et al. 2007, A\&A, 474, 827

Tanvir, N. R., Levan, A. J., Fruchter, A. S., et al. 2013, Natur, 500, 547

Tanvir, N. R., Levan, A. J., Fruchter, A. S., et al. 2015, GCN, 18100, 1

Tanvir, N. R., Levan, A. J., González-Fernández, C., et al. 2017, ApJL, 848, L27

Tauris, T. M., Kramer, M., Freire, P. C. C., et al. 2017, ApJ, 846, 170

Thielemann, F.-K., Arcones, A., Käppeli, R., et al. 2011, PrPNP, 66, 346

Troja, E., Butler, N., Watson, A. M., et al. 2017, GCN, 21051, 1

Troja, E., Sakamoto, T., Cenko, S. B., et al. 2016a, ApJ, 827, 102

Troja, E., Tanvir, N., Cenko, S. B., et al. 2016b, GCN, 20222, 1

Tunnicliffe, R. L., Levan, A. J., Tanvir, N. R., et al. 2014, MNRAS, 437, 1495

Updike, A. C., Bryngelson, G., \& Milne, P. A. 2009, GCN, 9361, 1

Villar, V. A., Guillochon, J., Berger, E., et al. 2017, ApJL, 851, L21

von Kienlin, A., Meegan, C., Goldstein, A., et al. 2017, GCN, 21520, 1

Yang, B., Jin, Z.-P., Li, X., et al. 2015, NatCo, 6, 7323 